\title{
Critical Roles of IL-33/ST2 Pathway in Neurological Disorders
}

\author{
Mohammad Faruq Abd Rachman Isnadi, ${ }^{1}$ Voon Kin Chin $\mathbb{D}^{2}$, Roslaini Abd Majid, $^{2}$ \\ Tze Yan Lee, ${ }^{3}$ Maizaton Atmadini Abdullah, ${ }^{3}$ Ramatu Bello Omenesa, ${ }^{1}$ \\ Zaid Osamah Ibraheem, ${ }^{1}$ and Rusliza Basir $\left(\mathbb{D}{ }^{1}\right.$ \\ ${ }^{1}$ Department of Human Anatomy, Faculty of Medicine \& Health Sciences, Universiti Putra Malaysia, Serdang, Malaysia \\ ${ }^{2}$ Department of Medical Microbiology \& Parasitology, Faculty of Medicine \& Health Sciences, Universiti Putra Malaysia, \\ Serdang, Malaysia \\ ${ }^{3}$ Department of Pathology, Faculty of Medicine \& Health Sciences, Universiti Putra Malaysia, Serdang, Malaysia
}

Correspondence should be addressed to Rusliza Basir; rusliza@upm.edu.my

Received 24 August 2017; Accepted 6 December 2017; Published 4 February 2018

Academic Editor: Ulrich Eisel

Copyright (c) 2018 Mohammad Faruq Abd Rachman Isnadi et al. This is an open access article distributed under the Creative Commons Attribution License, which permits unrestricted use, distribution, and reproduction in any medium, provided the original work is properly cited.

Interleukin-33 (IL-33) is an IL-1 family member, which exhibits both pro- and anti-inflammatory properties solely based on the type of the disease itself. Generally, IL-33 is expressed by both endothelial and epithelial cells and mediates its function based on the interaction with various receptors, mainly with ST2 variants. IL-33 is a potent inducer for the Th2 immune response which includes defence mechanism in brain diseases. Thus, in this paper, we review the biological features of IL-33 and the critical roles of IL-33/ST2 pathway in selected neurological disorders including Alzheimer's disease, multiple sclerosis, and malaria infection to discuss the involvement of IL-33/ST2 pathway during these brain diseases and its potential as future immunotherapeutic agents or for intervention purposes.

\section{Introduction}

IL-33, also designated as IL-1F11, is a newly identified cytokine which belongs to and is classified as the 11th member of the IL1 family [1]. It was first identified as DVS27, a highly upregulated gene found in canine vasospastic vertebral arteries [2], and later as the nuclear factor from high endothelial venules, NF-HEV [3], prior computational analysis by Schmitz and coworkers, which leads to the reidentification as IL-33 due to the sheer similarity with IL-1 family members [1]. IL-33 initiates its signalling pathway by binding to the ST2 receptor, or also known as IL-1R4, with variants which are comprised of ST2L, sST2, ST2V, and ST2LV [1, 4-7]. Apart from ST2, the interaction of IL-33 with a single immunoglobulin IL-1Rrelated molecule (SIGIRR) has been documented as well $[8,9]$. With exception from other IL-1 family members, IL-33 is primarily involved in the Th2 immune response induction [1] which confers protective immune response towards parasitic infection, specifically helminths [10-12], and deleterious immune response towards hypersensitivity or allergic reactions, especially asthma $[5,13,14]$.

IL-33 and its role has been widely investigated in numerous diseases particularly but not limited to the various degrees of hypersensitivity-related and infectious diseases, whereby several of the diseases are confined within the brain [15-17]. IL-33 is identified as a "dual cytokine" by exerting its protective and deleterious effect, either as a pro- or anti-inflammatory role, and based on acting as a regular cytokine or nuclear transcription factor, which depends upon the model and the disease [18] and type of cells $[1,19-22]$, hence appearing to be a "double-edged sword" [23]. There are several insights that point out the potential of IL-33/ST2 pathway for novel biomarker discovery and therapeutic target in diverse brain diseases $[16,24,25]$. Therefore, in this review, the critical roles of the IL-33/ST2 pathway in neurological disorders are discussed, particularly in Alzheimer's disease, multiple sclerosis, and experimental cerebral malaria. 


\section{Biological Features and Structure of IL-33}

IL-33 is a cytokine which exerts its dual functions on two mechanisms: either as an extracellular "traditional cytokine" or is considered as "intracellular nuclear factor with transcriptional regulatory properties" [26]. IL-33 mRNA or protein can be found in various types of organs by a multitude of cells including endothelial and certain epithelial cells $[1,20]$, mast cells [21], activated macrophages, and dendritic cells [1]. As a cytokine, IL-33 binds to ST2L/IL1-RAcP complex by inducing Th2 cytokines and chemokines through polarised Th2 cells, such as alternatively activated macrophages (AAM or M2) [27-32]. Despite the functional role of IL-33 as a nuclear transcription factor is yet to be fully elucidated, however, as a nuclear factor, the full length of IL-33 is confined in the nucleus of epithelial and endothelial cells $[3,20,33,34]$, consists of up to 270 amino acids, and possesses a homeodomain-like helix-turn-helix DNAbinding domain at the amino-terminal part, the $\mathrm{N}$ terminus, which is functional in nuclear localisation, heterochromatin association in the nucleus, and a repressor of transcriptional-based activities upon binding to the chromatin $[3,33,35]$. Furthermore, at the amino terminus part, the full length of IL-33 is also able to interact with the nuclear factor kappa-light-chain-enhancer of activated $\mathrm{B}$ cell $(\mathrm{NF}-\kappa \mathrm{B})$ transcription factors, p65 subunit [36]. This binding can be enhanced by IL-1 $\beta$ in the cytosol and nucleus, which in turn repress the gene activation by reducing the binding to other targets such as tumour necrosis factor alpha (TNF- $\alpha$ ) and nuclear factor of kappa light polypeptide gene enhancer in B cell inhibitor, alpha $(\mathrm{I} \kappa \mathrm{B} \alpha)$. Besides, IL-33 has been described in deterring regulatory gene transcription by binding with the heterochromatin-associated transcriptional repressor histone lysine methyltransferase SUV39HI [33] and/or acidic pocket of nucleosome of $\mathrm{H} 2 \mathrm{~A}-\mathrm{H} 2 \mathrm{~B}$ which induces the nucleosome-nucleosome interaction $[35,37]$.

IL-33 mediates its proinflammatory properties through the formation of IL-33/ST2L/IL1-RAcP complex [1, 27-29, 38] while inactivated through the conjugation of IL-33, sST2 [5], and SIGIRR $[8,9,39]$ or even cleaved by caspases during apoptosis [40-42]. Although it is noted that IL-33 acts as either pro- or anti-inflammatory cytokine, it is also suggested that IL-33 acts as a complex cytokine with features that also appears to be opposing both pro- and antiinflammatory cytokine as well $[43,44]$. IL-33 itself is thought to be as a pro-inflammatory inducer involved in the activation of various cells in the innate immune system, however, recent studies have suggested that IL-33 signalling associated with regulatory $\mathrm{T}$ cell (Treg) and regulatory B cell responses $[43,45]$. Additionally, another evidence points out the involvement of IL-33 expressed by the brain without an inflammatory response [46]. Mainly, IL-33 induces Th2 innate immune responses as compared with other IL-1 family members [1], though also potentially induces production of Th1 cytokine, IFN- $\gamma$ [30, 47]. Despite this, IL-33 solely acts as a chemoattractant for Th2 cells as opposed to Th1 cells [48]. IL-33 also has been described as an important amplifier for innate rather than adaptive immunity solely
[49], which certainly points out as crucial for both innate and adaptive immunity $[18,22,44,50,51]$. And in a certain point, IL-33 is being described as "alarmin" due to the cytokine involvement during necrosis and therefore is also associated with damage-associated membrane protein (DAMP) [26].

IL-33 is classified as one of the members of the IL-1 superfamily due to the sheer similarities of structures based on the $\beta$-trefoil structure with other members of IL-1 cytokines based on small-angle X-ray-scattering (SAXS) and X-ray crystallography analysis (Figure 1) $[32,52]$. IL-33 possesses 12 stranded $\beta$-trefoil fold, where these $12 \beta$-strands form the $\beta$-trefoil fold and conserved in both structures, while conformational variation residue in the loops containing the strain, chiefly $\beta 2-\beta 3, \beta 3-\beta 4, \beta 4-\beta 5, \beta 10-\beta 11$, and $\beta 11-\beta 12$ is involved in the receptor interaction. These 12 $\beta$-strands from the $\beta$-trefoil folds are arranged in 3 pseudorepeats of $4 \beta$-strands units, where the first and last $\beta$-strands are anti-parallel staves in a 6 -stranded $\beta$-barrel. Meanwhile, each repeat form of the second and third $\beta$-strands forms a $\beta$-hairpin sitting atop of the $\beta$-barrel. As a comparison, IL33 is identical to IL-18 based on the homologue of the amino acid sequence rather than with other IL-1 family members [1]. Additionally, IL-33 is confined in the nucleus of stromal cells such as IL- $1 \alpha$ as compared with other IL- 1 family members as well [53].

The full-length IL-33 is mainly expressed by stromal, epithelial, and endothelial cells (Figure 2) [1, 20]. Unlike IL-18 and IL-1 $\beta$, IL-33 does not require proteolytic processing by caspases-1, as a protease, in order to induce signalling via ST2L. Moreover, cleavage activity of caspase-3/7 towards IL-33 deactivates IL-33 and its bioactivities [40, 41]. Unlike Th1 cells, ST2 is expressed by Th2 cells when exposed to IL-33, followed by the secretion of IL- 5 and IL- 13 by Th2 cells $[1,10,54,55]$. IL-33-treated mast cells express IL-4, IL-5, and IL-16 [21,56] while IL-33-treated keratinocytes express IL- 6 and TNF- $\alpha$ [57].

\section{Releases and Signalling of IL-33}

In the event of necrosis, the release of the full-length IL-33 binds to the heterodimer receptor complex of ST2L/IL1RAcP, which consists of ST2L (T1, Fit-1, and DER4), a member of the Toll-like receptor (TLR)/IL1R superfamily [1, 27-29]. IL-33 does not form a heterodimer complex with ST2 directly, but rather IL-33 signalling is initiated through the recruitment of an accessory and a coreceptor protein, IL-1R accessory protein (IL1-RAcP), which is necessary to induce ligand-based signalling activation via the dimerisation of both ST2 and IL1-RAcP [29]. There are variants of ST2 receptors, namely, ST2L, a membrane-anchored receptor that responds to IL-33 by heterodimerising with IL-1RAcP $[4,58]$; sST2, a decoy and soluble isoform receptor identical to ST2L $[29,59]$; ST2V, an isoform receptor similar to sST2 with exception of the third extracellular immunoglobulin domain and can be found in human $[6,60]$; and ST2LV, similar with ST2L without transmembrane domain and currently described in chicken, Gallus gallus, solely [7]. Signalling is initiated by IL-1RAcP domain via Tollinterleukin-1 receptor (TIR) which leads to the recruitment 


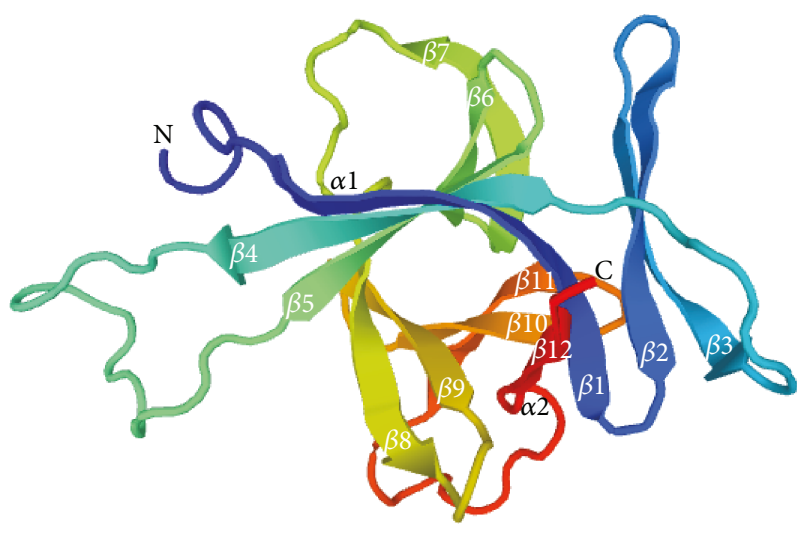

(a)

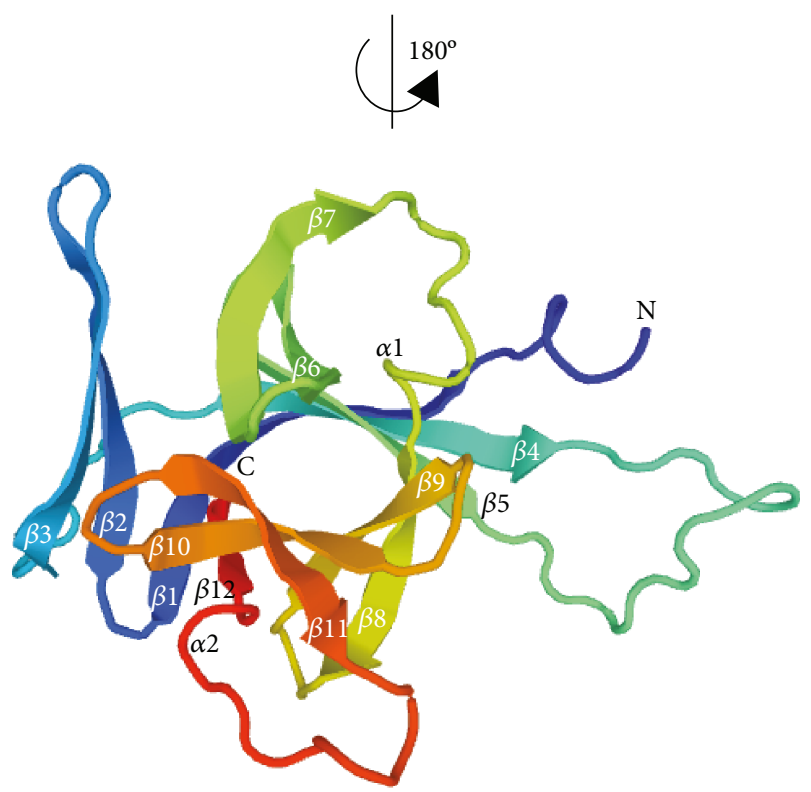

(b)

Figure 1: 3D molecular structure of IL-33. IL-33 represented in a ribbon diagram with labelled secondary structures. Note the arrangement of the $\beta$-strands from $\beta 1$ to $\beta 12$ that are arranged from the $\mathrm{N}$ - until C-terminus tail (a). (b) The view of (a) correlated by a $180^{\circ}$ rotation of the $y$-axis. IL-33 also acts as a ligand which binds to ST2, a high-affinity receptor family member [53]. A ternary complex formation is formed through the IL-33/ ST2 complex formation with IL-1RAcP. Furthermore, the designated binding area is somewhat to be both polar and nonpolar regions, which facilitate specific binding between a ligand and a receptor.

of MyD88 and subsequently IL-1R-associated kinase 1 or 4 (IRAK1/4) and TRAF6 [1, 61, 62]. TRAF6 further induces signalling of the mitogen-associated protein kinases (MAPK) and IKK pathways, which act as inducers and activate numerous inflammatory mediators and transcription factors such as P38, c-Jun N-terminal kinases (JNK), extracellular signal-regulated kinases (ERK), and NF- $\kappa \mathrm{B}$ mediators. These mediators and transcription factors finally drive the production of numerous Th2 cytokines and chemokines [1, 54, 63], which can be derived from several mechanisms. The first mechanism is through the regulation of the gene expression and modulation of chromatin via conjugation of the intracellular IL-33 with the acidic pocket of nucleosome of H2A$\mathrm{H} 2 \mathrm{~B}[35,37]$. This is achieved through the nuclear IL-33, which is acting as a regulatory transcriptional factor by downregulating the expression of certain receptors and cytokines such as soluble IL-1R4 and IL-6 through the conjugation with transcriptional repressor histone methyltransferase SUV39H [33]. Meanwhile, the second mechanism involves the downregulation of NF- $\kappa \mathrm{B}$ and proinflammatory signalling via conjugation of the intracellular IL-33 with NF$\kappa \mathrm{B}$ transcription factors [36].

In the meantime, the binding of IL-33 to sST2 further forms the IL-33/sST2 complex, which sST2 neutralises, and blocks the IL-33 inflammatory activities, thus repressing the NF- $\kappa \mathrm{B}$ and Th2 proinflammatory productions $[5,64]$. The soluble form, sST2, has been demonstrated to be elevated in several cardiac diseases and therefore considered as a biological marker for the prognosis of the events of cardiac diseases $[38,65,66]$. Inactivation of IL-33 also occurs through the inverse regulation of ST2L signalling by SIGIRR. Meanwhile, in the event of apoptosis, the releases of IL-33 is cleaved and further inactivated by caspase-3/7 via proteolysis [40-42].

\section{Roles of IL-33/ST2 Pathway in Neurological Disorders}

The involvement of IL-33/ST2 pathway has been incriminated in numerous diseases and conditions, such as in asthma and myocardial infarction. However, there are few studies that correlated with the participation of the IL-33/ ST2 pathway in the brain, specifically, neurological disorders involving the central nervous system (CNS). ST2L/IL-1RAcP and IL-33 are highly expressed in brain tissues, where IL-33 is constitutively expressed by endothelial cells, as well as by astrocytes, neurones, microglia, and oligodendrocytes while ST2L/IL-1RAcP by microglia, astrocytes, and neurones $[1,17,67]$. Meanwhile, sST2 is expressed by microglia and astrocytes [67]. IL-33 mRNA level was also revealed to be highly expressed in the spinal cord and brain of the mouse [1]. Exposure of microglia with IL-33 produces numerous proinflammatory cytokines such as TNF- $\alpha$, IL- $1 \beta$, and IL-10 and chemokines including CCL2, CCL3, CCL5, and CXCL10 and oxidative stress molecules, namely, nitric oxide (NO) and inducible nitric oxide synthase (iNOS) [67]. Notable and distinct involvement of IL-33/ST2 in neurological disorders is greatly reflected in Alzheimer's disease, multiple sclerosis, and experimental cerebral malaria as summarized in Table 1.

\section{Alzheimer's Disease}

Alzheimer's disease (AD) is a type of neurological disorder characterised by the progressive of cognitive impairment, memory deficits, and personality changes, and this disease causes rapid neurone loss and deteriorations; hence, the aetiology is still unknown and definite treatment is still unavailable [68-71]. Pathophysiologically, it is a form of dementia due to the excessive deposition of extracellular 


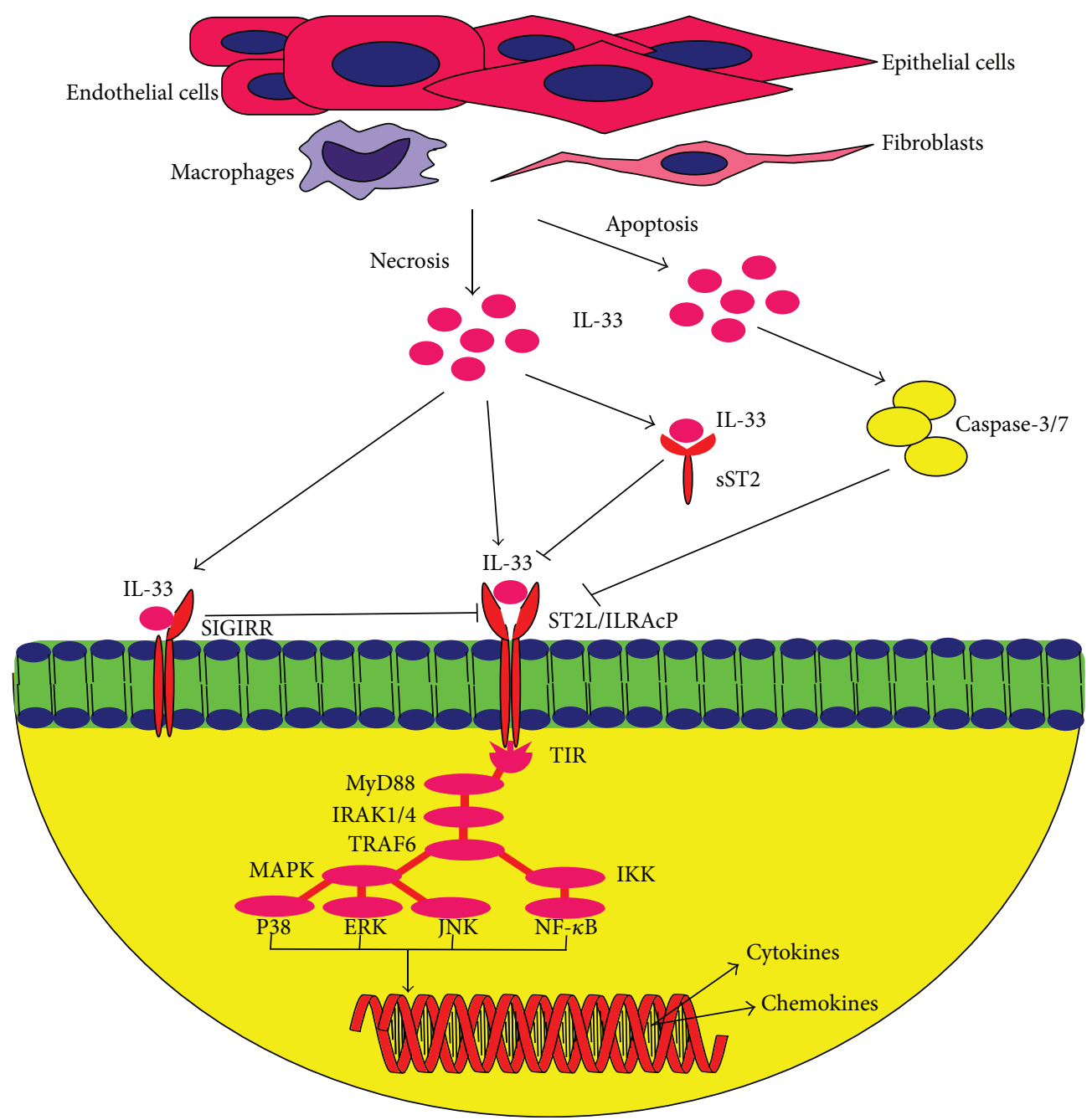

FIGURE 2: Releases and signalling of IL-33. Full length of IL-33 is predominantly expressed by stromal cells, endothelial and epithelial cells, macrophages, and fibroblasts. In necrosis, IL-33 is released and binds to ST2L/IL1-RAcP heterodimer receptor, which induces signalling via TIR domain of ST2L/IL1-RAcP and recruits MyD88 and followed by IRAK1/4 and TRAF6. TRAF6 further induces either MAPK which in turns activating P38, ERK and JNK, and/or IKK pathway which activate NF- $\kappa$ B. Both pathways lead to the recruitment of transcription factors and proinflammatory cytokines, which consequently drives the regulatory transcription factors of Th2 cytokines and chemokines. Alternatively, binding of IL-33 to sST2 neutralises the proinflammatory effect of IL-33 as well as with SIGIRR which negatively regulates ST2L signalling pathway. In apoptosis, IL-33 is cleaved and deactivated by caspase-3/7.

$\beta$-amyloid $(\beta A)$ plaque and intracellular tau-based neurofibrillary tangles (NFTs) in the brain [72]. Expression of IL-33 mRNA and protein was found to be significantly increased by pathogen-associated molecular patterns (PAMPs) in CNS glia and astrocytes, as well as IL-33 was stimulated and amplified by mast cells which directly induces numerous immune effectors in CNS glia such as arginase I, CCL17, CCL11, and TNF- $\alpha$ [19]. Transcriptomic analysis revealed the decrease in IL-33 expression in the brain of Alzheimer's disease cases and this expression is restricted to the endothelium and vascular smooth muscle cells of cell arteries of both $\mathrm{AD}$ and non-AD brains [73]. Additionally, overexpression of IL-33 was demonstrated in decreasing the $\mathrm{A} \beta_{40}$ peptide secretion, which is one of the main components for the cerebral amyloid angiopathy. Administration of IL-33 induces the proliferation of microglia and fosters the proinflammatory cytokine production such as TNF- $\alpha$ and
IL- $1 \beta$ and anti-inflammatory cytokine IL-10 and the production of chemokines CCL2, CCL3, CCL5, and CXCL10 and phagocytosis of nitric oxide by microglia [67]. High level of ST2 and IL-33 expressions was found to be localised in $\beta$ A plaques, in NFTs, and in the glial cells along with the increase of ST2 and IL-33-positive cells which suggest the involvement of IL-33 as an inflammatory inducer in the case of $\mathrm{AD}$ [72]. Intriguingly, the administration and modulation of IL-33 were found to alleviate the neuropathology of $\mathrm{AD}$ in mice by reducing the soluble $\beta \mathrm{A}$ levels and plaque accumulation through the recruitment of $\beta$ A phagocytic activity and the proinflammatory responses involving of IL- $1 \beta$, IL6 , and NLR family pyrin domain containing 3 (NLRP3) through the polarisation of macrophages/microglia [16]. These further suggest that IL-33 plays a crucial role in the pathogenesis of Alzheimer's disease as a mediator of inflammatory molecules. 
TABLE 1: Involvement of IL-33/ST2 pathways in variants of CNS diseases.

\begin{tabular}{|c|c|c|}
\hline Disorder & Findings & References \\
\hline \multirow{10}{*}{ Alzheimer's disease } & (i) IL-33 mRNA and protein expression was increased by PAMPs in CNS glia. & [19] \\
\hline & $\begin{array}{l}\text { (ii) IL-33-stimulated and IL-33-amplified mast cells directly induce arginase I, CCL17, CCL11, } \\
\& \text { TNF- } \alpha \text { in CNS glia. }\end{array}$ & \multirow{4}{*}{ [73] } \\
\hline & (iii) IL-33 expression is reduced in the brains. & \\
\hline & (iv) IL-33 decreases the secretion of $\mathrm{A} \beta_{40}$ peptides when overexpressed. & \\
\hline & $\begin{array}{l}\text { (v) Dose-dependent IL-33 induces proliferation of microglia and stimulates the production of IL- } 1 \beta \text {, } \\
\text { TNF- } \alpha \text {, \& IL- } 10 \text {. }\end{array}$ & \\
\hline & $\begin{array}{l}\text { (vi) IL-33 enhanced the production of CCL2, CCL3, CCL5, CXCL10, nitric oxide, and phagocytic } \\
\text { activity of microglia. }\end{array}$ & [67] \\
\hline & $\begin{array}{l}\text { (vii) IL-33 and ST2 expression was highly expressed in } \beta \text { A plaque, NFTs, and glial cells in the } \\
\text { AD brains. }\end{array}$ & \multirow[t]{2}{*}{ [72] } \\
\hline & (viii) IL-33 and ST2 ${ }^{+}$cells increased in the brain. & \\
\hline & (ix) IL-33 reduced soluble $\beta$ A levels and plaque accumulation via $\beta$ A phagocytic activity. & \multirow[b]{2}{*}{ [16] } \\
\hline & $\begin{array}{l}\text { (x) IL-33 modulation polarised microglia/macrophages and reduced IL-1 } \beta \text {, IL-6, \& NLRP3 } \\
\text { in the brain. }\end{array}$ & \\
\hline \multirow{8}{*}{ Multiple sclerosis } & (i) IL-33 was highly expressed in peripheral blood and CNS and plague areas in MS brains. & {$[80,81]$} \\
\hline & (ii) IL-33 plasma level was elevated compared with controls. & \multirow{2}{*}[81]{} \\
\hline & (iii) IL-33 plasma level was reduced in MS patients treated with IFN- $\beta$-1a. & \\
\hline & (iv) Intracellular IL-33 and OAS1, GATA3, \& PMAIP1 were highly expressed. & \multirow{3}{*}[79]{} \\
\hline & (v) IL-33 and HDAC were highly induced in RRMS patients. & \\
\hline & (vi) IL-33 might be regulating gene expression in RRMS. & \\
\hline & $\begin{array}{l}\text { (vii) IL-33 and ST2 expressions were enhanced in the lesions of acute and chronic MS patients } \\
\text { compared to healthy controls. }\end{array}$ & \multirow[t]{2}{*}[17]{} \\
\hline & (viii) IL-33 might inhibit CNS myelination. & \\
\hline \multirow{6}{*}{$\begin{array}{l}\text { Experimental cerebral } \\
\text { malaria }\end{array}$} & (i) IL-33 induces ILC2 expansion via IL-4, IL-5, \& IL-13 and polarises M2 macrophages and Tregs. & \multirow[b]{2}{*}[24]{} \\
\hline & $\begin{array}{l}\text { (ii) IL-33-treated } \mathrm{Treg}^{-/-} \text {mice were unable to resist ECM; IL-33-treated mice reduced parasitaemia } \\
\text { at the early phase of infection. }\end{array}$ & \\
\hline & (iii) $\mathrm{ST}^{-/-}$mice and WT mice exhibited no changes in parasitaemia and brain parasite load. & \multirow{3}{*}{ [15] } \\
\hline & (iv) $\mathrm{ST}^{-/-}$mice survived $>20$ days compared with WT mice with features of ECM. & \\
\hline & $\begin{array}{l}\text { (v) ICAM-1, CXCR3, and LT- } \alpha \text { expression reduced and diminished both } \mathrm{CD} 4^{+} \text {and } \mathrm{CD} 8^{+} \mathrm{T} \text {-cell } \\
\text { recruitment and sequestration. }\end{array}$ & \\
\hline & $\begin{array}{l}\text { (vi) IL-33 } 3^{-1-} \text { and WT mice exhibited no prominent changes in parasitaemia and survival rate. } \\
\text { (vii) TSLP mRNA expression was significantly decreased as compared to IL- } 33 \text { and IL-25 at the late } \\
\text { stage of infection. }\end{array}$ & [89] \\
\hline
\end{tabular}

\section{Multiple Sclerosis}

Multiple sclerosis (MS) is an autoimmune disease interfering with central nervous system (CNS) and is considered as chronic inflammatory-demyelinating disease due to the involvement of both adaptive and innate immunity responses [74-76]. It is demonstrated that the lesions in MS are comprised of numerous types of leukocytes which are incriminated to be the key factor contributing to the lesion formation [77, 78]. Nevertheless, specific biochemical components involved in leading to the event of MS are yet to be fully understood [79]. In a study utilising experimental autoimmune encephalitis (EAE) as an animal model for MS, IL33 and ST2 were found to be highly expressed in the spinal cord tissue, while ST2 expression was found to be highly elevated in the spinal cord tissue of EAE-induced mice [80]. EAE has also exacerbated in $\mathrm{ST}^{-/-}$mice as compared with
WT mice, while IL-33-treated WT mice developed impaired EAE. Additionally, it was demonstrated the increases of $\mathrm{CD} 4^{+} \mathrm{Foxp}^{+}$Treg cells were induced by IL-33 in the lymph nodes and CNS which further suggests the impairment of EAE perhaps due to the Treg cell induction through M2 macrophage induction by IL-33. Furthermore, adoptive transfer experiment of IL-33-treated macrophages impaired the severity of EAE in vivo. Meanwhile, the IL-33 level was found to be upregulated in the peripheral and central nervous system of MS patients, and IL-33 plasma level was found to be increased in MS patients as compared with controls; however, IL-33 blood level was found to be significantly decreased in MS patients treated with IFN- $\beta$-1a [81]. Additionally, IL-33 expression was highly increased in white matter and plaque areas of MS brains. The expression of intracellular IL-33 and IL-33-regulated genes such as OAS1, GATA3, and PMAIP1 was increased in relapsing remitting 
MS patients (RRMS) [79]. In vitro cultivation with TLR agonist lipopolysaccharide (LPS) further demonstrated the increase induction of IL-33 and histone deacetylase (HDAC), an enzyme that is involved in inflammatory regulation, in RRMS patients. Additionally, gene expression from the peripheral blood mononucleated cell (PBMC) culture treated with IL-33 was overlapped with RRMS patients, suggesting that the gene expression observed in RRMS was perhaps regulated by IL-33-mediated immune pathways. An EAE model was also performed and able to demonstrate the significant reduction of intracellular IL-33 as compared with the high increase of extracellular IL-33 in the naive mice during the preonset, onset, and peak stage of EAE in the spinal cord [25]. The damages of neurones and activation of astrocytes are associated with the reduction of intracellular IL-33 upon in vitro inflammatory stimulation during EAE. Additionally, EAE development was exacerbated through the blockage of CNS-derived IL-33 which further suggests the involvement of CNS-derived IL-33 in impairing EAE development. Meanwhile, in both cortex of MS patients and healthy controls, IL33 was found to be expressed by neurones, astrocytes, microglia, and oligodendrocytes while ST2 was expressed by oligodendrocytes and damaged axons [17]. Intriguingly, the expression of ST2 and IL-33 levels was enhanced in the brain lesions of both acute and chronic MS patients as compared with healthy controls. This further demonstrated the possible inhibition of CNS myelination by IL-33 from established rat CNS-myelinating cocultures, though the involvement of IL-33 in the neurodegenerative process still remains unclear. These evidences suggest that IL-33/ST2 pathway could be involved in the pathogenesis of MS or protective effect against MS.

\section{Experimental Cerebral Malaria}

Malaria is a devastating parasitic infection caused by protozoan Plasmodium parasites, transmitted by female Anopheles mosquitoes and is endemic in tropical and subtropical areas [82]. According to the World Health Organization (WHO) report, in 2015, approximately 214 million cases and up to 500,000 cases of mortality accounted worldwide with $88 \%$ of the cases were comprised of African regions alone [83]. There are many complications manifested due to malaria including cerebral malaria (CM), which is most commonly associated with Plasmodium falciparum ( $P$. falciparum) malaria infection that often leads to mortality [84]. Pathophysiology of CM is commonly associated with the sequestration of parasitised red blood cells (PRBCs) and inflammatory exudates in the microvessels, along with the exacerbated inflammation in the brain [84-87]. Experimental cerebral malaria (ECM) utilising murine Plasmodium berghei ( $P$. berghei) ANKA strain $(\mathrm{PbA})$ is widely utilised as a model for the human P. falciparum malaria [86-88]. In an ECM study, induction of IL-33 has caused the expansion of type 2 innate lymphoid cells (ILC2), through the type 2 cytokines, such as IL-13, IL-4, and IL-5, and has led to the polarisation of the M2 macrophages and Foxp3 Treg cells [24]. Additionally, Treg-deleted mice treated with IL-33 were unable to resist ECM, while IL-33-treated mice demonstrated significant parasitaemia reduction at the early phase of infection as compared with the untreated mice; however, they succumb to death at the later stage due to the hyperparasitaemia. Meanwhile, no changes of parasitaemia and brain parasite load were found between ST2-deficient and WT mice [15]. The ST2-deficient mice were able to survive more than 20 days without ECM features as compared with the WT mice which succumbed to death at day 10 with ECM, features, such as haemorrhages, brain vascular leakage, and distinct microvascular pathology obstruction. Additionally, the expression of CXCR3, ICAM-1, and LT- $\alpha$ was reduced which diminished recruitment and intravascular activation of both activated $\mathrm{CD}^{+}$and cytotoxic $\mathrm{CD} 8^{+} \mathrm{T}$-cells which subsequently promote ECM development. Furthermore, recent literature revealed that there were no significant changes in parasitaemia and survival rate between the mutant (IL-25 ${ }^{-1-}$, IL-33 ${ }^{-1-}$, and TSLP receptor, TSLPR ${ }^{-/-}$) and WT mice [89]. The expression of TSLP mRNA was found to be significantly decreased at the late stage of infections compared with mRNA expression of IL-33 and IL-25 in the brain. Thus, these contradictory findings warrant further investigations as the role mediated by IL-33 in ECM is still unclear at the moment to reflect CM.

\section{Conclusion}

IL-33 has been perceived to render either protective or deleterious role as an important Th2 cytokine inducer in numerous diseases; however, particular role(s) in CNS diseases are yet to be fully understood. It appears that IL-33 renders as a critical inducer and regulator of gene transcriptions and cytokines in innate and adaptive immunity either as an intracellular or extracellular cytokine. In necrosis, the IL-33/ST2 pathway has been demonstrated to be a critical signalling pathway inducing related immune mechanisms, while IL-33/sST2 appeared to be an interesting pathway in neutralising the activation of IL-33. Therefore, further studies are required to elucidate these pathways as they might be beneficial targets in CNS diseases either as novel biomarkers, therapeutic targets, or even as intervention purposes.

\section{Conflicts of Interest}

The authors declare that they have no conflicts of interest.

\section{References}

[1] J. Schmitz, A. Owyang, E. Oldham et al., "IL-33, an interleukin-1-like cytokine that signals via the IL-1 receptorrelated protein ST2 and induces T helper type 2-associated cytokines," Immunity, vol. 23, no. 5, pp. 479-490, 2005.

[2] H. Onda, H. Kasuya, K. Takakura et al., "Identification of genes differentially expressed in canine vasospastic cerebral arteries after subarachnoid hemorrhage," Journal of Cerebral Blood Flow \& Metabolism, vol. 19, no. 11, pp. 1279-1288, 1999.

[3] E. S. Baekkevold, M. Roussigné, T. Yamanaka et al., "Molecular characterization of NF-HEV, a nuclear factor preferentially 
expressed in human high endothelial venules," The American Journal of Pathology, vol. 163, no. 1, pp. 69-79, 2003.

[4] K. Yanagisawa, T. Takagi, T. Tsukamoto, T. Tetsuka, and S. Tominaga, "Presence of a novel primary response gene ST2L, encoding a product highly similar to the interleukin 1 receptor type 1," FEBS Letter, vol. 318, no. 1, pp. 83-87, 1993.

[5] H. Hayakawa, M. Hayakawa, A. Kume, and S. Tominaga, "Soluble ST2 blocks interleukin-33 signaling in allergic airway inflammation," Journal Biological Chemistry, vol. 282, no. 36, pp. 26369-26380, 2007.

[6] T. Si, K. Kuroiwa, K. Tago, H. Iwahana, K. Yanagisawa, and N. Komatsu, "Presence and expression of novel variant form of ST2 gene product in human leukemic cell line UT-7/GM," Biochemical and Biophysical Research Communications, vol. 264, no. 1, pp. 14-18, 1999.

[7] H. Iwahana, M. Hayakawa, K. Kuroiwa et al., "Molecular cloning of the chicken ST2 gene and a novel variant form of the ST2 gene product. ST2LV," Biochimica et Biophysica Acta (BBA)-Gene Structure and Expression, vol. 1681, no. 1, pp. 1-14, 2004.

[8] C. Garlanda, H. J. Anders, and A. Mantovani, "TIR8/SIGIRR: an IL-1R/TLR family member with regulatory functions in inflammation and T cell polarization," Trends in Immunology, vol. 30, no. 9, pp. 439-446, 2009.

[9] K. Bulek, S. Swaidani, J. Qin et al., “The essential role of single Ig IL-1 receptor-related molecule/Toll IL-1R8 in regulation of Th2 immune response," The Journal of Immunology, vol. 182, no. 5, pp. 2601-2609, 2009.

[10] D. R. Neill, S. H. Wong, A. Bellosi et al., "Nuocytes represent a new innate effector leukocyte that mediates type-2 immunity," Nature, vol. 464, no. 7293, pp. 1367-1370, 2010.

[11] H. J. McSorley, N. F. Blair, K. A. Smith, A. N. McKenzie, and R. M. Maizels, "Blockade of IL-33 release and suppression of type 2 innate lymphoid cell responses by helminth secreted products in airway allergy," Mucosal Immunology, vol. 7, no. 5, pp. 1068-1078, 2014.

[12] C. M. Finlay, A. M. Stefanska, K. P. Walsh et al., "Helminth products protect against autoimmunity via innate type 2 cytokines IL-5 and IL-33, which promotes eosinophilia," The Journal of Immunology, vol. 196, no. 2, pp. 703-714, 2016.

[13] D. Préfontaine, J. Nadigel, F. Chouiali et al., "Increased IL-33 expression by epithelial cells in bronchial asthma," The Journal of Allergy and Clinical Immunology, vol. 125, no. 3, pp. 752$754,2010$.

[14] H. Y. Lee, C. K. Rhee, J. Y. Kang et al., "Blockade of IL-33/ST2 ameliorates airway inflammation in a murine model of allergic asthma," Experimental Lung Research, vol. 40, no. 2, pp. 66-76, 2014.

[15] J. Palomo, F. Reverchon, J. Piotet et al., "Critical role of IL-33 receptor ST2 in experimental cerebral malaria development," European Journal of Immunology, vol. 45, no. 5, pp. 13541365, 2015.

[16] A. K. Fu, K. W. Hung, M. Y. Yuen et al., "IL-33 ameliorates Alzheimer's disease-like pathology and cognitive decline," Proceedings of the National Academy of Sciences of the United States of America, vol. 113, no. 19, pp. E2705-E2713, 2016.

[17] D. Allan, K. J. Fairlie-Clarke, C. Elliott et al., "Role of IL-33 and ST2 signalling pathway in multiple sclerosis: expression by oligodendrocytes and inhibition of myelination in central nervous system," Acta Neuropathologica Communications, vol. 4, no. 1, article 75, 2016.
[18] A. M. Miller, "Role of IL-33 in inflammation and disease," Journal of Inflammation, vol. 8, no. 1, p. 22, 2011.

[19] C. A. Hudson, G. P. Christophi, R. C. Gruber, J. R. Willmore, D. A. Lawrence, and P. T. Massa, "Induction of IL-33 expression and activity in central nervous system glia," Journal of Leukocyte Biology, vol. 84, no. 3, pp. 631-643, 2008.

[20] C. Moussion, N. Ortega, and J. P. Girard, "The IL-1-like cytokine IL-33 is constitutively expressed in the nucleus of endothelial cells and epithelial cells in vivo: a novel "alarmin"?," PLoS One, vol. 3, no. 10, article e3331, 2008.

[21] C. L. Hsu, C. V. Neilsen, and P. J. Bryce, "IL-33 is produced by mast cells and regulates IgE-dependent inflammation," PLoS One, vol. 5, no. 8, article e11944, 2010.

[22] M. De la Fuente, T. T. MacDonald, and M. A. Hermoso, "The IL-33/ST2 axis: role in health and disease," Cytokine \& Growth Factor Reviews, vol. 26, no. 6, pp. 615-623, 2015.

[23] R. Saluja, M. E. Ketelaar, T. Hawro, M. K. Church, M. Maurer, and M. C. Nawijin, "The role of the IL-33/IL-1RL1 axis in mast cell and basophil activation in allergic disorders," Molecular Immunology, vol. 63, no. 1, pp. 80-85, 2014.

[24] A. G. Besnard, R. Guabiraba, W. Niedbala et al., "IL-33mediated protection against experimental cerebral malaria is linked to induction of type 2 innate lymphoid cells, M2 macrophages and regulatory T cells," PLoS Pathogens, vol. 11, no. 2, article e1004607, 2015.

[25] H. Chen, Y. Sun, L. Lai et al., "Interleukin-33 is released in spinal cord and suppresses experimental autoimmune encephalomyelitis in mice," Neuroscience, vol. 308, pp. 157-168, 2015.

[26] G. Haraldsen, J. Balogh, J. Pollheimer, J. Sponheim, and A. M. Küchler, "Interleukin-33 - cytokine of dual function or novel alarmin?," Trends in Immunology, vol. 30, no. 5, pp. 227 233, 2009.

[27] A. A. Chackerian, E. R. Oldham, E. E. Murphy, J. Schmitz, S. Pflanz, and R. A. Kastelein, "IL-1 receptor accessory protein and ST2 comprise the IL-33 receptor complex," The Journal of Immunology, vol. 179, no. 4, pp. 2551-2555, 2007.

[28] S. Ali, M. Huber, C. Kollewe, S. C. Bischoff, W. Falk, and M. U. Martin, "IL-1 receptor accessory protein is essential for IL-33induced activation of T lymphocytes and mast cells," Proceedings of the National Academy of Sciences of the United States of America, vol. 104, no. 47, pp. 18660-18665, 2007.

[29] G. Palmer, B. P. Lipsky, M. D. Smithgall et al., "The IL-1 receptor accessory protein $(\mathrm{AcP})$ is required for IL-33 signalling and soluble AcP enhances the ability of soluble ST2 to inhibit IL-33," Cytokine, vol. 42, no. 3, pp. 358-364, 2008.

[30] M. D. Smithgall, M. R. Comeau, B. R. Yoon, D. Kaufman, R. Armitage, and D. E. Smith, "IL-33 amplifies both Th1-and Th2-type responses through its activity on human basophils, allergen-reactive Th2 cells, iNKT and NK cells," International Immunology, vol. 20, no. 8, pp. 1019-1030, 2008.

[31] M. Kurowska-stolarska, B. Stolarski, P. Kewin et al., "IL-33 amplifies the polarization of the alternatively activated macrophages that contribute to airway inflammation," The Journal of Immunology, vol. 183, no. 10, pp. 6469-6477, 2009.

[32] X. Liu, M. Hammel, Y. He et al., "Structural insights into the interaction of IL-33 with its receptors," Proceedings of the National Academy of Sciences of the United States of America, vol. 110, no. 37, pp. 14918-14923, 2013.

[33] V. Carriere, L. Roussel, N. Ortega et al., "IL-33, the IL-1-like cytokine ligand for ST2 receptor, is a chromatin-associated 
nuclear factor in vivo," Proceedings of the National Academy of Sciences of the United States of America, vol. 104, no. 1, pp. 282-287, 2007.

[34] A. M. Küchler, J. Pollheimer, J. Balogh et al., "Nuclear interleukin-33 is generally expressed in resting endothelium but rapidly lost upon angiogenic or proinflammatory activation," The American Journal of Pathology, vol. 173, no. 4, pp. 1229-1242, 2008.

[35] L. Roussel, M. Erard, C. Cayrol, and J. P. Girard, "Molecular mimicry between IL-33 and KSHV for attachment to chromatin through the $\mathrm{H} 2 \mathrm{~A}-\mathrm{H} 2 \mathrm{~B}$ acidic pocket," $E M B O$ Reports, vol. 9, no. 10, pp. 1006-1012, 2008.

[36] S. Ali, A. Mohs, M. Thomas et al., "The dual function cytokine IL-33 interacts with the transcription factor NF- $\kappa$ B to dampen NF- $\kappa \mathrm{B}$-stimulated gene transcription," The Journal of Immunology, vol. 187, no. 4, pp. 1609-1616, 2011.

[37] A. A. Kalashnikova, M. E. Porter-Goff, U. M. Muthurajan, K. Luger, and J. C. Hansen, "The role of the nucleosome acidic patch in modulating higher order chromatin structure," Journal of the Royal Society Interface, vol. 10, no. 82, article 20121022, 2013.

[38] S. Sanada, D. Hakuno, L. J. Higgins, E. R. Schreiter, A. N. McKenzie, and R. T. Lee, "IL-33 and ST2 comprise a critical biochemically induced and cardioprotective signaling system," The Journal of Clinical Investigation, vol. 117, no. 6, pp. 15381549, 2007.

[39] J. Qin, Y. Qian, J. Yao, C. Grace, and X. Li, "SIGIRR inhibits interleukin-1 receptor-and toll-like receptor 4mediating signaling through different mechanisms," Journal of Biological Chemistry, vol. 280, no. 26, pp. 25233-25241, 2005.

[40] C. Cayrol and J. P. Girard, "The IL-1-like cytokine IL-33 is inactivated after maturation by caspase-1," Proceedings of the National Academy of Sciences of the United States of America, vol. 106, no. 22, pp. 9021-9026, 2009.

[41] A. U. Lüthi, S. P. Cullen, E. A. McNeela et al., "Suppression of interleukin-33 bioactivity through proteolysis by apoptopic caspases," Immunity, vol. 31, no. 1, pp. 84-98, 2009.

[42] T. Ohno, K. Oboki, N. Kajiwara et al., "Caspase-1, caspase8 , and calpain are dispensable for IL-33 release by macrophages," The Journal of Immunology, vol. 183, no. 12, pp. 7890-7897, 2009.

[43] S. Sattler, G. S. Ling, D. Xu et al., "IL-10-producing regulatory $B$ cells induced by IL-33 (Breg ${ }^{\text {IL-33) }}$ effectively attenuate mucosal inflammatory responses in the gut," Journal of Autoimmunity, vol. 50, pp. 107-122, 2015.

[44] J. Lu, J. Kang, C. Zhang, and X. Zhang, "The role of IL-33/ST2L signals in the immune cells," Immunology Letters, vol. 164, no. 1, pp. 11-17, 2015.

[45] C. Schiering, T. Krausgruber, A. Chomka et al., "The alarming IL-33 promotes regulatory T-cell function in the intestine," Nature, vol. 513, no. 7519, pp. 564-568, 2014.

[46] G. Wicher, E. Husic, G. Nilsson, and K. Forsberg-Nilsson, "Developmental expression of IL-33 in the mouse brain," Neuroscience Letters, vol. 555, pp. 171-176, 2013.

[47] M. Komai-Koma, E. Wang, M. Kurowska-Stolarska, D. Li, C. McSharry, and D. Xu, "Interleukin-33 promoting Th1 lymphocyte differentiation dependents on IL-12," Immunobiology, vol. 221, no. 3, pp. 412-417, 2016.

[48] M. Komai-Koma, D. Xu, Y. Li, A. N. McKenzie, I. B. McInnes, and F. Y. Liew, "IL-33 is a chemoattractant for human Th2 cells," European Journal of Immunology, vol. 37, no. 10, pp. 2779-2786, 2007.

[49] K. Oboki, T. Ohno, N. Kajiwara et al., "IL-33 is a crucial amplifier of innate rather than acquired immunity," Proceedings of the National Academy of Sciences of the United States of America, vol. 107, no. 43, pp. 18581-18586, 2010.

[50] C. M. Lloyd, "IL-33 family members and asthma - bridging innate and adaptive immune responses," Current Opinion in Immunology, vol. 22, no. 6, pp. 800-806, 2010.

[51] N. T. Martin and M. U. Martin, "Interleukin 33 is a guardian of barriers and a local alarming," Nature Immunology, vol. 17, no. 2, pp. 122-131, 2016.

[52] A. Lingel, T. M. Weiss, M. Niebuhr et al., "Structure of IL-33 and its interaction with the ST2 and IL-1RAcP receptors insight into heterotrimeric IL-1 signaling complexes," Structure, vol. 17, no. 10, pp. 1398-1410, 2009.

[53] P. Rider, Y. Carmi, E. Voronov, and R. N. Apte, "Interleukin$1 \alpha$," Seminars in Immunology, vol. 25, no. 6, pp. 430-438, 2013.

[54] M. Kurowska-Stolarska, P. Kewin, G. Murphy et al., "IL-33 induces antigen-specific IL- $5^{+} \mathrm{T}$ cells and promotes allergicinduced airway inflammation independent of IL-4," The Journal of Immunology, vol. 181, no. 7, pp. 4780-4790, 2008.

[55] J. Louten, A. L. Rankin, Y. Li et al., "Endogeneous IL-33 enhances Th2 cytokine production and T-cell responses during allergic airway inflammation," International Immunology, vol. 23, no. 5, pp. 307-315, 2011.

[56] D. Xu, H. R. Jiang, P. Kewin et al., "IL-33 exacerbates antigeninduced arthritis by activating mast cells," Proceedings of the National Academy of Sciences of the United States of America, vol. 105, no. 31, pp. 10913-10918, 2008.

[57] P. Li, H. Ma, D. Han, and K. Mou, "Interleukin-33 affects cytokine production by keratinocytes in vitiligo," Clinical and Experimental Dermatology, vol. 40, no. 2, pp. 163-170, 2015.

[58] H. Li, K. Tago, K. Io et al., "The cloning and nucleotide sequence of human ST2L cDNA," Genomics, vol. 67, no. 3, pp. 284-290, 2000.

[59] D. A. Pascual-Fiqal and J. L. Januzzi, "The biology of ST2: the international ST2 consensus panel," The American Journal of Cardiology, vol. 115, no. 7, pp. 3B-7B, 2015.

[60] K. Tago, T. Noda, M. Hayakawa et al., "Tissue distribution and subcellular localization of a variant form of the human ST2 gene product, ST2V," Biochemical and Biophysical Research Communications, vol. 285, no. 5, pp. 1377-1383, 2001.

[61] M. Funakoshi-Tago, K. Tago, M. Hayakawa et al., "TRAF6 is a critical signal transducer in IL-33 signaling pathway," Cellular Signalling, vol. 20, no. 9, pp. 1679-1686, 2008.

[62] M. U. Martin, "Special aspects of interleukin-33 and the IL-33 receptor complex," Seminars in Immunology, vol. 25, no. 6, pp. 449-457, 2013.

[63] M. Funakoshi-Tago, S. Y. Tago-K, S. Tominaga, and T. Kasahara, "JAK2 is an important signal transducer in IL33 -induced NF- $\kappa \mathrm{B}$ activation," Cellular Signalling, vol. 23, no. 2, pp. 363-370, 2011.

[64] C. Garlanda, F. Riva, E. Bonavita, and A. Mantovani, "Negative regulatory receptors of the IL-1 family," Seminars in Immunology, vol. 25, no. 6, pp. 408-415, 2013.

[65] S. Demyanets, C. Kaun, R. Pentz et al., "Components of the interleukin-33/ST2 system are differentially expressed and regulated in human cardiac cells and in cells of the cardiac 
vasculature," Journal of Molecular and Cellular Cardiology, vol. 60, no. 100, pp. 16-26, 2013.

[66] J. Sánchez-Más, A. Lax, C. Asensio-López Mdel et al., "Modulation of IL-33/ST2 system in postinfarction heart failure: correlation with cardiac remodelling markers," European Journal of Clinical Investigation, vol. 44, no. 7, pp. 643-651, 2014.

[67] S. Yasuoka, J. Kawanokuchi, B. Parajuli et al., "Production and functions of IL-33 in the central nervous system," Brain Research, vol. 1385, pp. 8-17, 2011.

[68] M. D. Carter, G. A. Simms, and D. F. Weaver, "The development of new therapeutics for Alzheimer's disease," Clinical Pharmacology and Therapeutics, vol. 88, no. 4, pp. 475-486, 2010.

[69] G. M. McKhann, D. S. Knopman, H. Chertkow et al., "The diagnosis of dementia due to Alzheimer's disease: recommendations from the National Institute on Aging-Alzheimer's Association workgroups on diagnostic guidelines for Alzheimer's disease," Alzheimers Dement, vol. 7, no. 3, pp. 263-269, 2011.

[70] A. D. Korczyn, "Why we failed to cure Alzheimer's disease?," Journal of Alzheimer's Disease, vol. 29, no. 2, pp. 275-282, 2012.

[71] M. C. Carrillo, R. A. Dean, F. Nicolas et al., "Revisiting the framework of the National Institute on Aging-Alzheimer's Association diagnostic criteria," Alzheimer's \& Dementia, vol. 9, no. 5, pp. 594-601, 2013.

[72] Z. Xiong, R. Thangavel, D. Kempuraj, E. Yang, S. Zaheer, and A. Zaheer, "Alzheimer's disease: evidence for the expression of interleukin-33 and its receptor ST2 in the brain," Journal of Alzheimer's Disease, vol. 40, no. 2, pp. 297-308, 2014.

[73] J. Chapuis, D. Hot, F. Hansmannel et al., "Transcriptomic and genetic studies identify IL-33 as a candidate gene for Alzheimer's disease," Molecular Psychiatry, vol. 14, no. 11, pp. 1004-1016, 2009.

[74] J. H. Noseworthy, C. Lucchinetti, M. Rodriguez, and B. G. Weinshenker, "Multiple sclerosis," The New England Journal of Medicine, vol. 343, no. 13, pp. 938-952, 2000.

[75] E. M. Frohman, M. K. Racke, and C. S. Raine, "Multiple sclerosis - the plaque and its pathogenesis," The New England Journal of Medicine, vol. 354, no. 9, pp. 942-955, 2006.

[76] M. Sospedra and R. Martin, "Immunology of multiple sclerosis," Annual Review of Immunology, vol. 23, no. 1, pp. 683-747, 2005.

[77] C. Lucchinetti, W. Brück, J. Parisi, B. Scheithauer, M. Rodriguez, and H. Lassmann, "Heterogeneity of multiple sclerosis lesions: implications for the pathogenesis of demyelination," Annals of Neurology, vol. 47, no. 6, pp. 707-717, 2000.

[78] B. Kornek and H. Lassmann, "Neuropathology of multiple sclerosis - new concepts," Brain Research Bulletin, vol. 61, no. 3, pp. 321-326, 2003.

[79] F. Zhang, J. T. Tossberg, C. F. Spurlock, S. Y. Yao, T. M. Aune, and S. Sriram, "Expression of IL-33 and its epigenetic regulation in multiple sclerosis," Annals of Clinical Translational Neurology, vol. 1, no. 5, pp. 307-318, 2014.

[80] H. R. Jiang, M. Milovanović, D. Allan et al., "IL-33 attenuates EAE by supressing IL-17 and IFN- $\gamma$ production and inducing alternatively activated macrophages," European Journal of Immunology, vol. 42, no. 7, pp. 1804-1814, 2012.
[81] G. P. Christophi, R. C. Gruber, M. Panos, R. L. Christophi, B. Jubelt, and P. T. Massa, "Interleukin-33 upregulation in peripheral leukocytes and CNS of multiple sclerosis patients," Clinical Immunology, vol. 142, no. 3, pp. 308-319, 2012.

[82] L. French, T. Gray, and P. Natarajan, "Malaria: prevention and treatment," InnovAiT: Education and inspiration for general practice, vol. 7, no. 4, pp. 224-232, 2014.

[83] World Health Organization, World Malaria Report 2015, World Health Organization, Geneva, Switzerland, 2015.

[84] R. Idro, N. E. Jenkins, and C. R. Newton, "Pathogenesis, clinical features, and neurological outcome of cerebral malaria," The Lancet Neurology, vol. 4, no. 12, pp. 827-840, 2005.

[85] N. H. Hunt and G. E. Grau, "Cytokines: accelerators and brakes in the pathogenesis of cerebral malaria," Trends in Immunology, vol. 24, no. 9, pp. 491-499, 2003.

[86] A. G. Craig, G. E. Grau, C. Janse et al., "The role of animal models for research on severe malaria," PLoS Pathogens, vol. 8, no. 2, article e1002401, 2012.

[87] R. Basir, S. S. Fazalul Rahiman, K. Hasballah et al., "Plasmodium berghei ANKA infection in ICR mice as a model of cerebral malaria," Iran Journal of Parasitology, vol. 7, no. 4, pp. 62-74, 2012.

[88] J. B. de Souza and E. M. Riley, "Cerebral malaria: the contribution of studies in animal models to our understanding of immunopathogenesis," Microbes and Infection, vol. 4, no. 3, pp. 291-300, 2002.

[89] A. Shibui, A. Takamori, M. E. M. Tolba et al., "IL-25, IL-33 and TSLP receptor are not critical for development of experimental murine malaria," Biochemistry and Biophysics Reports, vol. 5, pp. 191-195, 2016. 


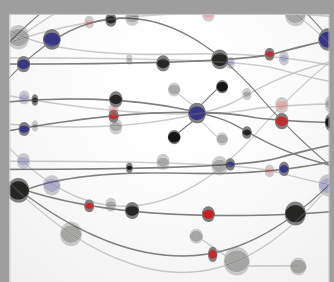

The Scientific World Journal
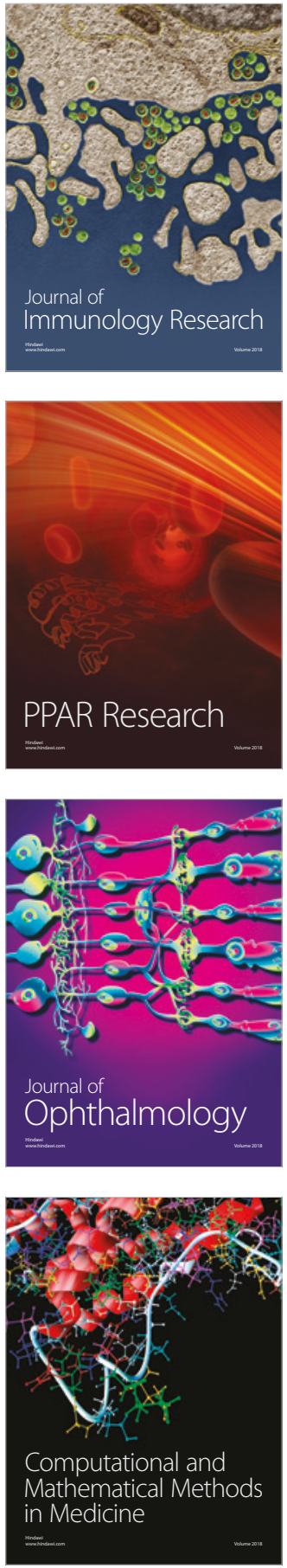

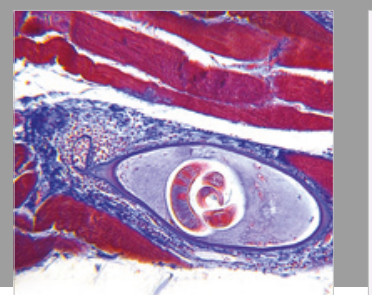

Gastroenterology Research and Practice

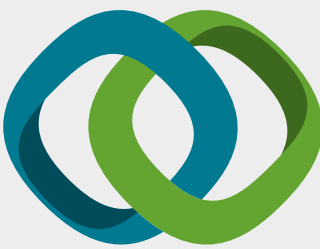

\section{Hindawi}

Submit your manuscripts at

www.hindawi.com
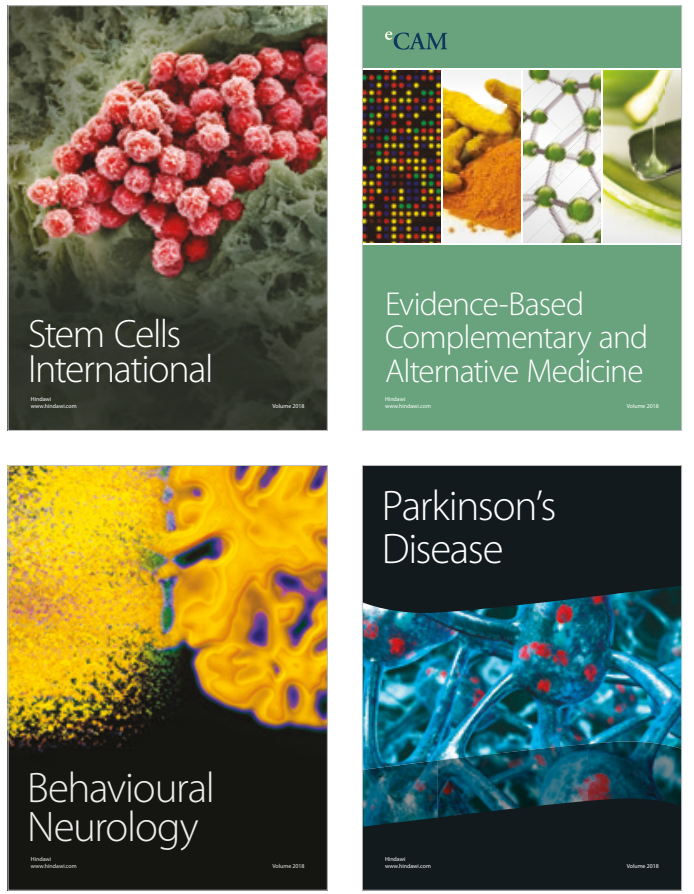

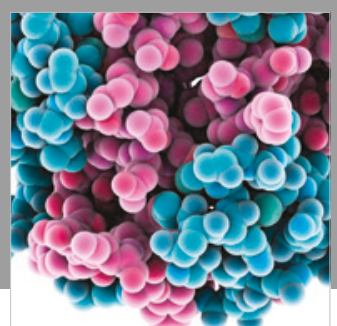

ournal of

Diabetes Research

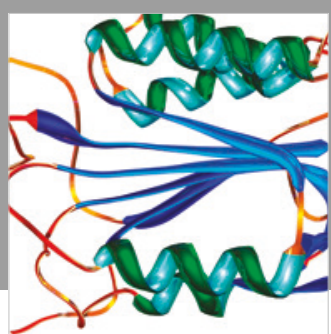

Disease Markers
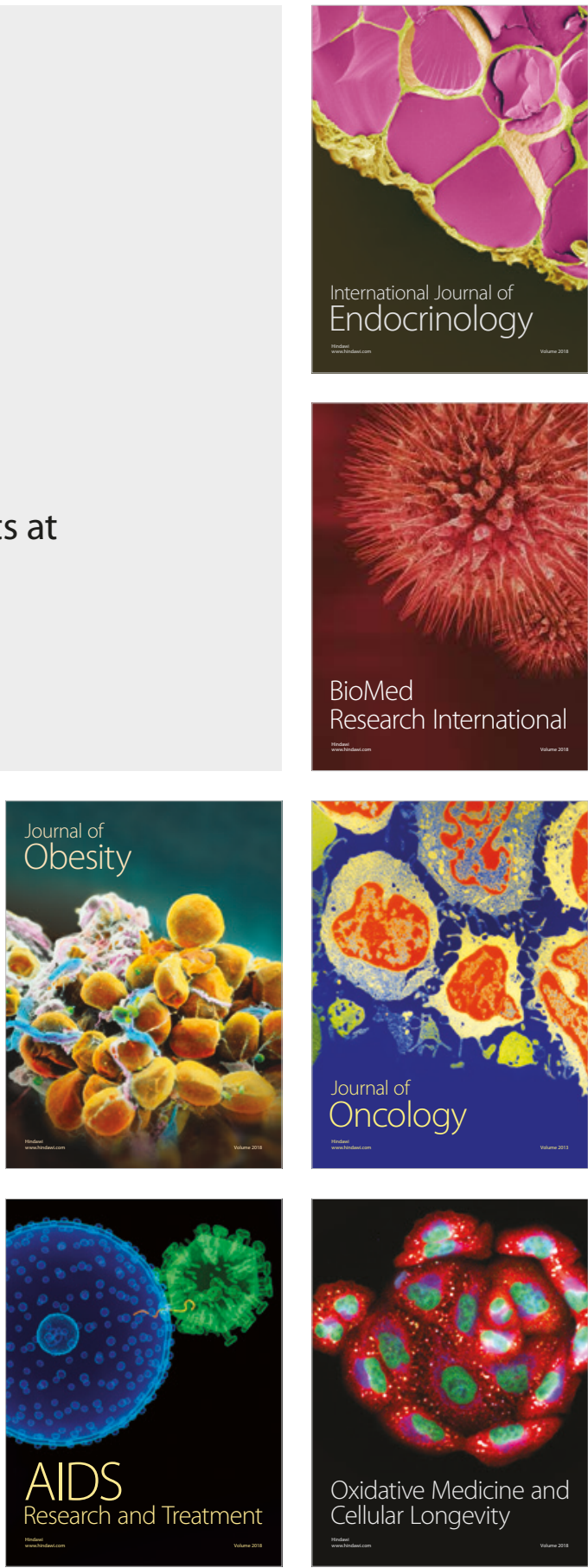